\title{
Quelles pistes pour sortir de l'impasse?
}

\section{Valérie Junod}

Professeure de droit aux universités de Genève et Lausanne

Le Tribunal fédéral a dernièrement tranché un cas de responsabilité médicale où le médecin mis en cause était accusé d'homicide par négligence (référence: 6B 1287/2018). Ce médecin généraliste, au bénéfice d'une longue pratique, avait omis de contrôler la fiche de traitement que le personnel de l'EMS, où était institutionnalisé son patient, un homme polymorbide de près de 80 ans, lui avait soumise pour vérification. En vue du renouvellement de la prescription, ce document indiquait les divers médicaments qui devaient être pris par le patient, soit une dizaine chaque jour! Regrettablement, à la suite d'une erreur, cette fiche indiquait que le patient devait prendre 2,5 mg de méthotrexate tous les jours, alors que la posologie correcte, suivie dans son cas depuis déjà des années, était d'une prise hebdomadaire. Cette erreur était due à une mauvaise communication et transcription lors du transfert du patient depuis son précédent EMS. On soulignera que le médecin, poursuivi ici pénalement, n’avait ni prescrit le méthotrexate, ni organisé le transfert, ni administré les médicaments, ni établi la fiche en cause. Il lui avait été uniquement demandé de contresigner cette dernière alors qu'il était de passage dans l'EMS pour prendre des nouvelles de son patient qui avait fait une chute. Néanmoins, si le médecin avait lu le document attentivement, il aurait décelé l'erreur et aurait indubitablement interrompu le traitement; le patient n'aurait alors ingéré qu'une double dose de méthotrexate. Quand l'erreur a finalement été découverte, le patient avait pris le médicament pendant cinq jours d'affilée et son état s'était fortement dégradé. Informé par le personnel de l'EMS, le médecin a alors instruit d'arrêter la prise de méthotrexate durant deux semaines et d'exercer une surveillance rapprochée sur le patient; il a en revanche renoncé à ordonner une hospitalisation immédiate. Celle-ci a néanmoins eu lieu en urgence le lendemain. Malgré les soins prodigués, le patient est décédé dix jours plus tard et le décès a été attribué à une surdose de méthotrexate.

\section{Arguments du Tribunal fédéral}

Dans la procédure pénale mise en œuvre, le médecin a d'abord été acquitté par le tribunal de première ins- tance neuchâtelois en février 2018. Sur appel, la Cour pénale neuchâteloise l'a condamné en novembre 2018 à 30 jours-amende à $300 \mathrm{CHF}$ le jour. Ayant fait recours, le Tribunal fédéral lui donne raison. Le médecin est donc définitivement libéré des charges qui pesaient sur lui. Ce dernier jugement du 11 mars 2019 est instructif à plusieurs égards.

D’abord, le Tribunal fédéral confirme que le médecin a commis un manquement aux règles de l'art médical. De l'avis du Tribunal, le médecin aurait dû vérifier le document soumis et un tel examen «même assez sommaire" aurait alors suffi à mettre à jour l'erreur, avec conséquence l'arrêt précoce du traitement erroné. Le Tribunal rappelle les principes qui gouvernent la responsabilité médicale. Le médecin répond de toute violation fautive des règles de prudence et règles de l'art, même si ce manquement n'est pas «grave». Toutefois, lorsque le médecin est confronté à une incertitude médicale, celle-ci conduit souvent à admettre une «latitude de jugement», de sorte que «le médecin ne viole [alors] son devoir de diligence que lorsqu'il pose un diagnostic ou choisit une thérapie ou une autre méthode qui, selon l'état général des connaissances professionnelles, n'apparaît plus défendable». Dans le cas d'espèce, le manquement reproché ne relevait pas d'une telle marge d'appréciation. Le Tribunal conclut donc sans difficultés à une violation du devoir de prudence en raison de l'inattention blâmable. Ce constat n'est pas inattendu.

Ensuite, et c'est là où l'arrêt devient intéressant, le Tribunal vérifie la condition de la causalité. Plus précisément, il s'interroge sur ce qui se serait passé si le médecin poursuivi avait détecté l'erreur après la deuxième prise, comme il l'aurait dû. A ce stade, le Tribunal se repose sur l'avis des experts médicaux qui avaient été mis en œuvre par le juge cantonal. En l'espèce, l'expert avait écrit: «l'issue fatale aurait pu, peut-être, être évitée». Or, en droit pénal [1], sous l'angle de la causalité hypothétique, pour que le médecin puisse être condamné pour son omission (à savoir le fait de n'avoir pas agi, ici de n'avoir pas vérifié attentivement le document et de n'avoir pas signalé l'erreur), il est nécessaire qu'avec «une très grande vraisemblance», l'acte at- 
tendu (i.e. signaler l'erreur) eût exclu le résultat (i.e. le décès du patient). Pour le Tribunal fédéral, la conclusion telle que formulée par l'expert ne satisfait pas ce standard, puisqu'il n'était justement pas très vraisemblable que le patient eût survécu (seul l'homicide était en cause) si le personnel soignant lui avait immédiatement administré «l'antidote».

Une des conditions exigées pour prononcer la condamnation pénale du médecin faisant défaut, ce dernier est acquitté.

\section{Que penser de cet arrêt?}

On y trouve «à boire et à manger».

D'un côté, les professionnels de la santé seront soulagés de savoir que, s'ils n'ont rien fait (omission d'agir alors qu'ils occupent une position de garant), ils ne seront condamnés pénalement que s'il est évident que leur action aurait empêché le résultat (ici le décès, mais plus souvent une lésion corporelle). Tant que les experts médicaux concluent que l'issue favorable pour le patient ne pouvait pas être prévue avec certitude en cas d'action corrective appropriée, les soignants échappent à la sanction. En pratique, en présence d'une omission, le pronostic sur des faits hypothétiques est toujours délicat. Est-ce que la maladie, le grand âge ou d'autres circonstances, notamment d'autres erreurs, n'auraient de toute façon pas conduit au décès, même si le soignant avait "mieux agi»? Les cas où l'arrièreplan médical est suffisamment clair et/ou l'expert suffisamment courageux ou sûr de lui pour affirmer que l'action différente de son collègue aurait permis d'éviter le décès ou la lésion devraient rester rares. De même que les cas de condamnations pénales.

Les professionnels de la santé pourraient s'inquiéter en réalisant que la moindre inattention peut engendrer des conséquences extrêmement lourdes.

De l'autre côté, les professionnels de la santé pourraient s'inquiéter en réalisant que la moindre inattention - ici lire trop vite une feuille récapitulant une dizaine de médicaments prescrits - peut engendrer des conséquences extrêmement lourdes allant jusqu'à la poursuite pénale pour homicide par négligence. Le médecin avait certainement supposé que cette feuille de traitement avait simplement été reprise du premier EMS au second, comme c'était usuellement le cas, puisque le problème de communication informatique entre les deux EMS ne lui avait pas été signalé. Le médecin avait dû jeter un regard aux noms des médicaments, mais manifestement ne s'était pas attaché à vérifier, pour chacun d'entre eux, la posologie exacte. D'ailleurs, on peut se demander si un médecin en déplacement dans un EMS pour rendre visite à son patient a, accessible à distance, le dossier médical complet de ce dernier, de manière à vérifier précisément la posologie.

Pour les patients, en particulier les patients ayant subi une erreur médicale, le constat est inversé. Ils se réjouiront d'apprendre que leur médecin traitant est censé vérifier soigneusement les prescriptions de médicaments, afin de pouvoir repérer les erreurs avant que celles-ci ne provoquent des atteintes à la santé. A l'inverse, ils réaliseront que leurs chances d'obtenir une condamnation pénale - le constat est quelque peu différent au civil - sont faibles. Face à une expertise médicale prudente (ce que devrait au demeurant être toute expertise), leurs chances sont mêmes quasi nulles.

\section{Alternative pour régler les litiges médicaux}

L'arrêt du 11 mars 2019 amène à s'interroger - et ce n'est pas la première fois - sur la nécessité d'un système alternatif pour régler les litiges médicaux. Il est de plus en plus largement admis [2], dans une perspective sociale, éthique et de contrôle-qualité, qu'un médecin n'ayant ni «fauté» intentionnellement ni commis de négligence impardonnable ne devrait pas encourir de poursuites pénales. En effet, aucun médecin, sur une carrière s'étendant sur 40 ans, n'est à l'abri d'une erreur ou d'un moment d'inattention. Sur le plan personnel, subir des années de procédure, surtout pénale, peut être extrêmement éprouvant pour le médecin, et ce quand bien même le médecin "gagne» à la fin. La simple perception d'une menace perpétuelle peut inciter les professionnels de la santé:

- à pratiquer une médecine dite défensive avec les coûts et les inconvénients que cela peut impliquer pour les patients et le système de santé;

- à fuir les patients "compliqués», en les renvoyant vers l'hôpital ou vers des médecins spécialisés;

- à se détourner des spécialisations dites à risque;

- à dissimuler leurs erreurs plutôt que de les discuter autour d'eux afin d'enseigner à leurs collègues à les éviter. A cet égard, on soulignera qu'un cas pratiquement identique avait déjà donné lieu à un arrêt du Tribunal fédéral du 6 mars 2007, sans que manifestement les enseignements nécessaires en aient été tirés (i.e. risques liés aux erreurs de transcription des prescriptions de méthotrexate chez le patient âgé). L'effet «éducatif» de la poursuite pénale laisse donc à désirer. 
La responsabilité pénale qui pèse presque exclusivement sur les individus "sur le terrain» peut en outre détourner des mesures qui devraient être prises au niveau des institutions, notamment pour mieux coordonner les soins et pour pallier les erreurs de systèmes (p. ex. en lien avec une surcharge chronique de travail dans certains services à une certaine période).

Peut-être, paradoxalement, pour le patient, la situation n'est guère meilleure. Le plus souvent, le patient et ses proches souhaitent obtenir une reconnaissance des souffrances subies, des excuses pour les erreurs ou les manquements commis et une indemnisation lorsqu'un dommage économique (p. ex. perte de gain) ou moral (p. ex. pour les douleurs et l'anxiété) est survenu. Ce n'est que lorsque l'attitude des soignants leur paraît inadmissible ou qu'il n'est guère commode d'agir par une autre voie que le patient privilégie la

\section{L'essentiel en bref}

- Le Tribunal fédéral a dernièrement acquitté un médecin accusé d'homicide par négligence pour ne pas avoir vérifié une fiche de traitement à la posologie erronée. Le praticien était en visite dans l'EMS de son patient (polymorbide et âgé).

- La cour reconnaît un manquement aux règles de l'art médical. Interrogés sur ce qui se serait passé si le médecin avait vu l'erreur au moment de contresigner la fiche, les experts estiment que la mort du patient «aurait pu, peut-être, être évitée». Une incertitude trop grande pour condamner le généraliste.

- Pour régler plus facilement les litiges médicaux, l'auteure propose un système moins lourd basé sur la médiation, la reconnaissance rapide des manquements et des indemnisations forfaitaires.

\section{Das Wichtigste in Kürze}

- Das Bundesgericht hat jüngst einen der fahrlässigen Tötung angeklagten Arzt freigesprochen, dem vorgeworfen wurde, eine Behandlungskarte nicht auf falsche Dosierung geprüft zu haben. Der Allgemeinmediziner war auf Visite bei seinem Patienten (polymorbid und betagt) im Alters- und Pflegeheim.

- Das Gericht stellte eine Nichtbeachtung der Regeln der Heilkunst fest. Befragt über das, was geschehen wäre, hätte der Arzt den Fehler bei der Gegenzeichnung der Karte bemerkt, schätzten die Experten, dass der Tod des Patienten «hätte, vielleicht, vermieden werden können". Zu viel Ungewissheit, um einen praktischen Arzt verurteilen zu können.

- Um medizinische Verfahren leichter regeln zu können, empfiehlt die Autorin ein leichteres, auf die Mediation, die schnelle Anerkennung von Fehlern/Versäumnissen und pauschalisierte Entschädigungen basiertes System. voie pénale. Si une alternative digne, pratique et rapide lui est proposée, par exemple une médiation avec une reconnaissance des fautes et un dédommagement à la clé, le patient a toutes les raisons d'opter pour cette voie.

A notre avis, le système actuel devrait être revu afin d'encourager de telles procédures extrajudiciaires. Il ne serait pas difficile d'introduire dans la loi une phase de médiation (par des organismes privés [3]) ou de conciliation spécialisées (par des organismes publics) obligatoire, avant que des procédures judiciaires civiles ou pénales ne puissent être entamées. Dans cette phase préalable, les pouvoirs d'enquête de ces organismes devraient être étendus, afin de s'assurer que les preuves et les témoignages utiles puissent être récoltés, avant qu'ils ne se perdent. Les délais de prescription seraient de plus suspendus. Les indemnisations devraient être calculées forfaitairement, par exemple à l'instar de ce qui existe dans le régime LAA (assurance-accidents) et est maintenant repris par la Fondation d'indemnisation des victimes de l'amiante. Le financement du système pourrait être semi-privé, un peu à l'image du système LAMal, les assureurs RC actuels ayant alors l'obligation de collaborer à ces procédures extrajudiciaires et de payer les montants alloués à leur terme.

Notre proposition aurait le mérite de permettre un règlement rapide et organisé de la plupart des litiges. Les professionnels de la santé n'auraient plus (ou en tout cas moins) la crainte d'une épée de Damoclès menaçant leur réputation et leur vie professionnelle et économique. Les patients auraient le confort d'être pris en charge par des spécialistes formés à ce genre de litige et capables d'accueillir leurs doléances et leurs souffrances.

\section{Références}

1 Le droit civil et le droit pénal divergent s'agissant du seuil de vraisemblance pour la causalité hypothétique en cas d'omission. En droit civil, l'exigence est plus basse, puisqu'il suffit que le lésé prouve la vraisemblance prépondérante (plus de 50\%). Cf. sur la causalité en droit civil: P. Ducor, L'expert médical et la causalité, in: Chappuis / Winiger (éds), Les causes du dommage: Journée de responsabilité civile 2016, Schulthess, 2007, p. 179-216. En l'espèce, le Tribunal fédéral aurait dû appliquer le standard de droit civil aux prétentions pour tort moral élevées par les proches du défunt.

2 Voir p. ex. R. Ameratunga et al., Criminalisation of unintentional error in healthcare in the UK: a perspective from New Zealand BMJ 2019:364.

3 On citera le travail remarquable effectué par le Bureau d'expertises extrajudiciaires de la FMH. Celui-ci n'est cependant pas compétent pour allouer une indemnisation, uniquement pour donner un avis sur l'existence d'un manquement aux règles de l'art. Voir les rapports annuels publiés depuis 2002 à partir du site de https:// www.fmh.ch/fr/a-propos-de-la-fmh/organisation/expertisesextrajudicaires.cfm 